\title{
Changes in Intracortical Excitability of Affected and Unaffected Hemispheres After Stroke Evaluated by Paired-Pulse Transcranial Magnetic Stimulation
}

\author{
Ho Youn Seo, $\mathrm{MD}^{1}$, Gi-Wook Kim, MD, $\mathrm{PhD}^{1,2}$, Yu Hui Won, $\mathrm{MD}, \mathrm{PhD}^{1,2}$, \\ Sung-Hee Park, MD, PhD ${ }^{1,2}$, Jeong-Hwan Seo, MD, $\mathrm{PhD}^{1,2}$, Myoung-Hwan Ko, MD, $\mathrm{PhD}^{1,2}$
}

${ }^{1}$ Department of Physical Medicine and Rehabilitation, Chonbuk National University Hospital, Chonbuk National University Medical School, Jeonju; ${ }^{2}$ Research Institute of Clinical Medicine of Chonbuk National UniversityBiomedical Research Institute of Chonbuk National University Hospital, Jeonju, Korea

Objective To assess the altered pattern of intracortical excitability of the affected and unaffected hemispheres in stroke patients using paired-pulse transcranial magnetic stimulation (TMS).

Methods We evaluated intracortical inhibition (ICI) and intracortical facilitation (ICF) in both hemispheres at acute and subacute stages of 103 stroke patients using paired-pulse TMS. The patients were divided into two groups: mild-to-moderate patients whose motor evoked potential (MEP) was recorded in the affected hemisphere; and severe patients whose MEP was not recorded in the affected hemisphere.

Results In mild-to-moderate patients, the value of ICI in the affected hemisphere was increased from $70.3 \%$ to $77.9 \%$ and the value of ICI in the unaffected hemisphere was decreased from $74.8 \%$ to $70.3 \%$ with eventual progression in acute to subacute stages of stroke. In severe patients, the value of ICI in the unaffected hemisphere was increased from $65.4 \%$ to $75.6 \%$. The changes in ICF were not significantly different in this study.

Conclusion We conclude that the unaffected hemisphere was more disinhibited than the affected hemisphere in acute phase of mild-to-moderate stroke, and the affected hemisphere was more disinhibited in the subacute stage. The unaffected hemisphere was inhibited in severe cases in acute-to-subacute phases of stroke. This finding facilitates appropriate neuromodulation of acute-to-subacute phases in mild-to-severe stroke patients.

Keywords Transcranial magnetic stimulation, Cortical excitability, Stroke

Received July 19, 2017; Accepted October 10, 2017

Corresponding author: Myoung-Hwan Ko

Department of Physical Medicine and Rehabilitation, Chonbuk National University Hospital, 20 Geonji-ro, Deokjin-gu, Jeonju 54907, Korea. Tel: +8263-250-1795, Fax: +82-63-254-4145, E-mail: mhko@jbnu.ac.kr

ORCID: Ho Youn Seo (http://orcid.org/0000-0002-2576-0749); Gi-Wook Kim (http://orcid.org/0000-0002-1628-8382); Yu Hui Won (http://orcid. org/0000-0003-2007-9652); Sung-Hee Park (http://orcid.org/0000-0002-4743-2551); Jeong-Hwan Seo (http://orcid.org/0000-0002-6915-6674); Myoung-Hwan Ko (http://orcid.org/0000-0002-0566-3677).

(c) This is an open-access article distributed under the terms of the Creative Commons Attribution Non-Commercial License (http://creativecommons.org/ licenses/by-nc/4.0) which permits unrestricted noncommercial use, distribution, and reproduction in any medium, provided the original work is properly cited. Copyright $\odot 2018$ by Korean Academy of Rehabilitation Medicine 


\section{INTRODUCTION}

Most patients with stroke do not show complete functional recovery and are limited in their activities of daily living for the rest of their life. Neurological recovery occurs as the neural networks within the cerebral cortex undergo changes in response to various environmental and physical stimuli. It may be accompanied by dissociation as tissue reorganization occurs as part of functional recovery, during brain plasticity [1]. Research into brain plasticity in patients with stroke is essential to elucidate the neurophysiological mechanisms of recovery from brain damage, and to develop better methods to modulate neural reorganization. Non-invasive neuromodulation techniques such as repetitive transcranial magnetic stimulation (TMS) and transcranial direct current stimulation have been conducted in patients with stroke. Despite several benefits [2-6], no results of specific stimulation or neuroanatomical targets along the phasic progression after stroke have been reported [7]. It is important to comprehend the degree of cortical excitability of each hemisphere in different phases of stroke for application of adequate neuromodulations.

Paired-pulse TMS is a non-invasive method of brain stimulation for the evaluation of cortical excitability in brain. In paired-pulse TMS, a sub-threshold conditioning stimulus is delivered at the primary motor cortex of the cerebral hemispheres before the supra-threshold test stimulus using several time interval protocols [8]. Responses are suppressed by application of conditioning stimulus at relatively short intervals of 1-6 ms. The test response is facilitated via application of conditioning stimulus at relatively long intervals of $10-15 \mathrm{~ms}$. This procedure is used to measure intracortical inhibition (ICI) and intracortical facilitation (ICF), which reflect excitability of short inhibitory and facilitatory interneuronal circuits within the motor cortex [8-10]. Studies correlated changes in intracortical excitability in the affected hemisphere and unaffected hemispheres, and motor function recovery is important as a neurophysiological marker in patients with stroke [10-12].

In a previous study with paired-pulse TMS, Manganotti et al. [10] reported that ICI was reduced in the unaffected hemisphere in the acute stage of stroke and ICI in the unaffected hemisphere returned to normal during the subacute phase of stroke.
However, studies using paired-pulse TMS investigating the changes in excitability of the cerebral cortex immediately after stroke are still limited by the lack of neuromodulation in different states of damaged brain. In the present study, to determine the state of cortical excitability in each hemisphere during acute-to-subacute phases of stroke, we observed changes in excitability of each cerebral cortex using paired-pulse TMS in patients with mild-to-severe stroke who underwent 3-week rehabilitation therapy.

\section{MATERIALS AND METHODS}

\section{Subjects}

This retrospective study was conducted using the medical records of patients with stroke who were admitted within 1 month of disease onset to the Department of Rehabilitation at Chonbuk University Hospital between March 2015 and February 2017. Inclusion criteria for patients enrolled in this study were: (1) a monohemipheric vascular lesion on CT or MRI, (2) presence of hemiplegia, and (3) a motor evoked potential (MEP) test and pairedpulse TMS before and after 3-week rehabilitation therapy. Exclusion criteria were: (1) previous stroke history, (2) lesions on both cerebral hemispheres or lesions in the cerebellum, (3) second stroke during the study period, and (4) other disorders, which affected extremity functions such as neuropathies or systemic vasculopathies.

\section{Evaluation methods with paired-pulse TMS}

The TMS system by MagPro (MagVenture, Lucernemarken, Denmark) and figure- 8 type magnetic coils (70 $\mathrm{mm}$ in diameter) were used to stimulate the motor cortex. A recording electrode was attached to the most prominent part of the 1st dorsal interosseous muscle, and electromyographic signals were digitalized in the form of amplitude and frequency [9]. The coils were placed on the scalp over the motor cortex parallel to each another. The optimal scalp position ('hot spot') was defined as the site, where the lowest stimulator output intensity consistently yielded the largest amplitude and minimal latency MEPs [10]. An electrical current was passed through the motor cortex hand area through the coils. The resting motor threshold intensity was defined as the lowest stimulator output intensity capable of inducing MEPs measuring at least $50 \mu \mathrm{V}$ in peak-to-peak amplitude in at least 
half of the 10 trials [13]. The intensity of the conditioning stimulus and the test stimulus were set to $80 \%$ and $120 \%$ of the resting motor threshold, respectively. These stimuli were alternately applied to the motor cortex containing the lesion. The mean amplitude of MEPs generated after the motor cortex was stimulated for 10 sessions was expressed as a percentage of the threshold value. The MEP values obtained at a stimulus interval of $3 \mathrm{~ms}$ were used as a parameter of ICI and those obtained at a stimulus interval of $10 \mathrm{~ms}$ were used as a parameter of ICF.

\section{Methods of assessment}

The patients were divided into two groups depending on whether MEP was observed on the affected hemisphere (group A) or not observed on the affected hemisphere (group B).

Among the patients in group A, the values of ICI and ICF from the affected and the unaffected hemispheres were measured during $\mathrm{T} 1$, i.e., the initial recording session (before rehabilitation), and $\mathrm{T} 2$, the second recording session (after rehabilitation). The values of ICI and ICF derived from only unaffected hemisphere were measured at $\mathrm{T} 1$ and $\mathrm{T} 2$ in group $\mathrm{B}$. To evaluate functional recovery, the Korean-Modified Barthel Index (K-MBI) and the Fugl-Meyer Assessment of upper extremity (FMA-UE) scores were measured at $\mathrm{T} 1$ and $\mathrm{T} 2$.

\section{Statistical analysis}

Statistical analyses were performed with SPSS version 18.0 (SPSS Inc., Chicago, IL, USA). The paired t-test was conducted to evaluate changes in intracortical excitability in each hemisphere in each group during the rehabilitation therapy as well as the K-MBI and FMA-UE. The independent t-test was used to compare intracortical excitability at $\mathrm{T} 1$ and $\mathrm{T} 2$. The correlation between the functional scores and values in excitability of the cerebral hemispheres in each group was analyzed with Pearson correlation. The level of significance was set at $\mathrm{p}<0.05$.

\section{RESULTS}

A total of 103 patients including 54 males and 49 females who met the aforementioned criteria were included in the study. Of the 103 patients, only 69 showed MEP in the affected hemisphere (Table 1). The mean age of the patients was $64.8 \pm 14.6$ years. The mean time from stroke to T1 was $14.8 \pm 12.1$ days, and the mean time from stroke to $\mathrm{T} 2$ was $36.8 \pm 16.7$ days. No statistically significant differences in sex and age of the patients were detected between the two groups.

In group A, the value of ICI significantly increased from $70.3 \% \pm 16.7 \%$ at $\mathrm{T} 1$ to $77.9 \% \pm 13.3 \%$ at $\mathrm{T} 2$ in the affected hemisphere $(\mathrm{p}=0.01)$, and significantly decreased from $74.8 \% \pm 14.2 \%$ at $\mathrm{T} 1$ to $70.3 \% \pm 15.0 \%$ at $\mathrm{T} 2$ in the unaffected hemisphere ( $\mathrm{p}=0.04)$ (Table 2, Fig. 1). The value of ICF decreased from $138.1 \% \pm 41.9 \%$ at $\mathrm{T} 1$ to $135.6 \% \pm 38.2 \%$ at $\mathrm{T} 2$ in the affected hemisphere, and from $130.4 \% \pm 28.4 \%$ at $\mathrm{T} 1$ to $126.5 \% \pm 22.0 \%$ at $\mathrm{T} 2$ in the unaffected hemisphere; these changes were not statistically significant. At T1, the value of ICI in the affected hemisphere was significantly smaller than in the unaffected hemisphere in group A $(p=0.01)$. However, at T2 the value of ICI in the affected hemisphere was significantly higher than in the unaffected hemisphere $(\mathrm{p}=0.04)$.

In group B, the value of ICI significantly increased from $65.4 \% \pm 14.4 \%$ at $\mathrm{T} 1$ to $75.6 \% \pm 13.1 \%$ at $\mathrm{T} 2$ in the unaffected hemisphere $(\mathrm{p}=0.01)$. The value of ICF decreased from $136.8 \% \pm 44.2 \%$ at $\mathrm{T} 1$ to $135.8 \% \pm 20.9 \%$ at $\mathrm{T} 2$, without any statistical significance.

The mean K-MBI score significantly increased from

Table 1. Patients' demographics and clinical characteristics

\begin{tabular}{|c|c|c|c|}
\hline & $\begin{array}{c}\text { Group A } \\
(n=69)\end{array}$ & $\begin{array}{c}\text { Group B } \\
(n=34)\end{array}$ & $\begin{array}{c}\text { Total } \\
(n=103)\end{array}$ \\
\hline Age (yr) & $67.7 \pm 13.9$ & $59.1 \pm 14.5$ & $64.8 \pm 14.6$ \\
\hline \multicolumn{4}{|l|}{ Gender } \\
\hline Male & 37 & 17 & 54 \\
\hline Female & 32 & 17 & 49 \\
\hline \multicolumn{4}{|c|}{ Affected hemisphere } \\
\hline Left & 37 & 15 & 52 \\
\hline Right & 32 & 19 & 51 \\
\hline \multicolumn{4}{|l|}{ Etiology } \\
\hline Infarction & 51 & 21 & 72 \\
\hline Hemorrhage & 18 & 13 & 31 \\
\hline \multicolumn{4}{|l|}{ Days after stroke } \\
\hline $\begin{array}{l}\text { 1st assessment } \\
\text { session (T1) }\end{array}$ & $12.1 \pm 11.5$ & $17.6 \pm 12.4$ & $14.8 \pm 12.1$ \\
\hline $\begin{array}{l}\text { 2nd assessment } \\
\text { session (T2) }\end{array}$ & $34.4 \pm 14.1$ & $41.8 \pm 20.3$ & $36.8 \pm 16.7$ \\
\hline
\end{tabular}

Values are presented as mean \pm standard deviation. 
Table 2. Parameters for intracortical excitability of cerebral hemisphere

\begin{tabular}{|c|c|c|c|c|c|c|}
\hline & \multicolumn{4}{|c|}{ Group A $(n=69)$} & \multirow{2}{*}{\multicolumn{2}{|c|}{$\begin{array}{c}\text { Group B }(n=34) \\
\text { UH }\end{array}$}} \\
\hline & \multicolumn{2}{|c|}{ AH } & \multicolumn{2}{|c|}{ UH } & & \\
\hline & T1 & T2 & T1 & T2 & T1 & T2 \\
\hline ICI (\%) & $70.3 \pm 16.7$ & $77.9 \pm 13.3^{*}$ & $74.8 \pm 14.2$ & $70.3 \pm 15.0^{*}$ & $65.4 \pm 14.4$ & $75.6 \pm 13.1^{*}$ \\
\hline $\operatorname{ICF}(\%)$ & $138.1 \pm 41.9$ & $135.6 \pm 38.2$ & $130.4 \pm 28.4$ & $126.5 \pm 22.0$ & $136.8 \pm 44.2$ & $135.8 \pm 20.9$ \\
\hline
\end{tabular}

Values are presented as mean \pm standard deviation.

$\mathrm{AH}$, affected hemisphere; UH, unaffected hemisphere; T1, 1st assessment session; T2, 2nd assessment session; ICI, intracortical inhibition; ICF, intracortical facilitation.

${ }^{*} \mathrm{p}<0.05$.

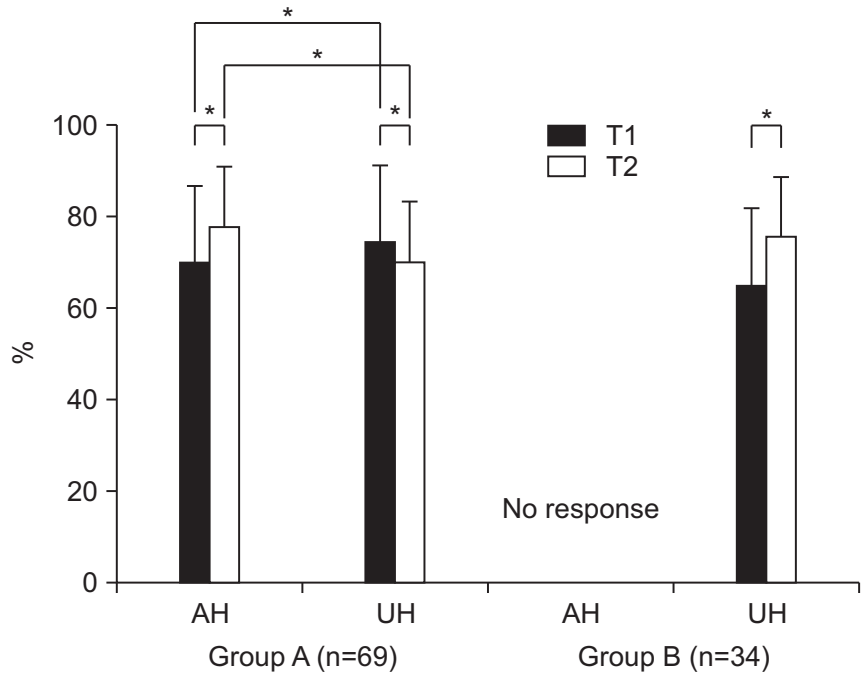

Fig. 1. Comparison of the changes in intracortical excitability of cerebral hemisphere. AH, affected hemisphere; UH, unaffected hemisphere; T1, 1st assessment session; $\mathrm{T} 2$, 2nd assessment session. ${ }^{*} \mathrm{p}<0.05$.

$53.3 \pm 23.3$ to $75.2 \pm 19.7(\mathrm{p}=0.01)$ in group $\mathrm{A}$, and from $21.7 \pm 21.4$ to $49.7 \pm 22.3(\mathrm{p}=0.01)$ in group $B$. The mean FMA-UE score significantly increased from $43.0 \pm 17.3$ to $52.7 \pm 12.9(\mathrm{p}=0.01)$ in group $\mathrm{A}$, and from $7.9 \pm 8.6$ to $11.1 \pm 11.4(\mathrm{p}=0.01)$ in group $\mathrm{B}$.

The changes in the mean K-MBI and FMA-UE scores were not significantly correlated with the changes in ICI and ICF in both groups (Table 3).

\section{DISCUSSION}

In the present study, we measured the excitability of intracortical hemispheres of mild-to-severe stroke patients in acute-to-subacute phase. Excitability of unaffected hemisphere is more disinhibited than that of affected hemisphere in the acute phase of mild-to-moderate stroke, whereas the excitability of affected hemisphere is more disinhibited in the subacute stage. Excitability in unaffected hemisphere increases with severity in acuteto-subacute phases of stroke.

Patients were divided into two experimental groups depending on whether or not MEPs were recorded in the ipsilesional hemisphere because the present study classified stroke severity based on electrophysiological properties rather than the functional status. Group A was more likely mild-to-moderate in stroke severity whereas group $\mathrm{B}$ was treated as a severe group.

In this study, the value of ICI was higher in the unaffected hemisphere than in the affected hemisphere in the acute phase of mild-to-moderate stroke. Manganotti et al. [10] proposed that 5-7 days after mild-to-moderate stroke, inhibition in the unaffected hemisphere was found to be accompanied by reduced inhibitory pathways in the affected hemisphere altered by GABAergic modulation and, accordingly, resulting in an inhibition imbalance in the two cerebral hemispheres [14]. One of the mechanisms demonstrated impairment in transcallosal inhibition and ipsilateral silent periods, indicating disinhibition [15]. When reduced motor inhibition induces unmasking of normally suppressed or inhibited pathways, the unaffected hemisphere is modulated functionally by activating cortico-cortical connections $[16,17]$. Excessive levels of inhibitory stimuli interfere with the reorganization around the damaged site, and limit the motor function recovery [18].

In the present study, approximately 5 weeks after stroke, the value of ICI in the affected hemisphere was higher than the ICI of the unaffected hemisphere in mild-tomoderate stroke. Manganotti et al. [10] reported that ICI in the unaffected hemisphere returned to normal level similar to that of healthy adults in 30 days after stroke in 
Table 3. Pearson correlation between changes of intracortical excitability and functional outcomes

\begin{tabular}{lcccccccc}
\hline & \multicolumn{3}{c}{ Group A } & & \multicolumn{2}{c}{ Group B } \\
\cline { 2 - 3 } \cline { 7 - 8 } & ICI & ICF & & ICI & UH & \multicolumn{2}{c}{ UH } \\
\hline K-MBI & $-0.032(0.797)$ & $-0.102(0.406)$ & & $0.078(0.524)$ & $-0.058(0.637)$ & & $-0.070(0.695)$ & $-0.103(0.564)$ \\
FMA-UE & $-0.078(0.522)$ & $-0.026(0.834)$ & & $0.050(0.685)$ & $0.035(0.774)$ & & $-0.071(0.688)$ & $-0.008(0.965)$ \\
\hline
\end{tabular}

Values are presented as $\mathrm{r}$ ( $\mathrm{p}$-value).

K-MBI, Korean-Modified Barthel Index; FMA-UE, Fugal-Meyer Assessment of upper extremity; AH, affected hemisphere; UH, unaffected hemisphere; ICI, intracortical inhibition; ICF, intracortical facilitation.

mild-to-moderate patients, without significantly altering the levels in patients with severe stroke. Cicinelli et al. [9] conducted paired-pulse stimulation $30.6 \pm 7.4$ days after mild-to-moderate stroke, and demonstrated that the value of ICI in the affected hemisphere was higher than in the unaffected hemispheres and in the hemispheres of healthy controls. This loss of inhibition in the affected hemisphere is considered to be a compensatory process, and it is presumed that the reduction of GABA activity facilitated cortical plasticity and optimized the recovery of motor functions [10-12]. The results were attributed to possible changes in the proportion of nerve sheath within the corticospinal tract that maintains the motor function after stroke [9]. Although this study supported the feasibility of this hypothesis, further studies investigating the long-term effects of disinhibition in the affected side are still needed.

In the study reported by Manganotti et al. [10], the value of ICI was not changed significantly in the unaffected hemispheres of patients with severe stroke even after a month of recovery. In the present study, which included a larger number of patients, the value of ICI in the unaffected hemisphere was increased after rehabilitation in patients with severe stroke. The damaged hemisphere does not fully recover, leading to an imbalance in excitability between the two cerebral hemispheres. The effect induces the unaffected hemisphere to constantly exert inhibitory stimuli on the affected hemisphere. This phenomenon appears to support the recovery paradigm that suggests a transfer of function from the unaffected to the affected hemisphere under conditions of diminished chances of recovery in the damaged brain area [19].

Several rodent studies reported that the number of NMDA receptors temporarily increases on the 5th day after brain damage $[14,20]$. In the study of Cicinelli et al. [9] comparing ICF between patients with stroke and healthy controls, no significant difference in ICF was found. No significant difference in the value of ICF was detected between the two cerebral hemispheres as the state of stroke changed from acute to subacute in the study of Manganotti et al [10]. Similarly, we observed no significant changes in ICF of patients with neither mild-to-moderate nor severe stroke.

While Honaga et al. [21] reported a correlation between motor function and changes in the level of ICI in patients with chronic hemiplegia and stroke, many studies failed to correlate normalization of ICI in the unaffected hemisphere with functional recovery $[9,10,12]$. Similarly, we found no significant correlations between the changes in intracortical excitability and altered K-MBI and FMA-UE scores. Intracortical excitability was not closely associated with functional recovery and further investigations are needed to corroborate this finding.

Variable methods of neuromodulation are based on targets of specific functional circuitry related to the degree of cortical excitability and movement activation. However, the optimal stimulation paradigms to maximize the benefits of rehabilitation are not established $[7,22]$. The present study should encourage further investigation using variable neuromodulation techniques in different phases of stroke.

In contrast to previous studies that mostly investigated a small number of patients $[9,10,12,14]$, the present study included a large number of patients. We observed changes in excitability of the affected and unaffected cerebral hemispheres over time according to the severity of stroke.

This study has several limitations. First, whether or not MEPs were recorded in the ipsilateral hemisphere may not reflect stroke severity. However, since the present study focused on electrophysiological changes induced by neurophysiological damage, it is unclear whether MEP was the primary parameter to assess stroke sever- 
ity. Second, since MEP evaluation and paired-pulse TMS examination were performed by several examiners, the methods of assessment used showed limited accuracy, and the reliability of cortical stimulation test results is questionable [23]. Finally, although it has been suggested, that the inhibitory circuits traverse the transcallosal pathway [12,15], we did not consider lesion location.

In conclusion, in patients with mild-to-moderate stroke, the unaffected hemisphere was more disinhibited than the affected hemisphere in the acute phase of stroke. The unaffected hemisphere was inhibited to a higher degree than the affected hemisphere during the subacute phase of stroke. Disinhibition of the unaffected cerebral hemisphere in patients with severe stroke was persistent at acute to subacute phases of stroke. According to this study, we expect that the changes in excitability of both hemispheres in patients with acute-to-subacute phases of mild-to-severe stroke facilitate our understanding of the recovery process and formulate an effective approach to neuromodulation in stroke.

\section{CONFLICT OF INTEREST}

No potential conflict of interest relevant to this article was reported.

\section{ACKNOWLEDGMENTS}

This research was supported by a grant awarded by the Korea Health Technology R\&D Project through the Korea Health Industry Development Institute, funded by the Ministry of Health \& Welfare, Republic of Korea (Grant No. HI15C1529).

\section{REFERENCES}

1. Sanes JN, Donoghue JP. Plasticity and primary motor cortex. Annu Rev Neurosci 2000;23:393-415.

2. Hummel F, Celnik P, Giraux P, Floel A, Wu WH, Gerloff C, et al. Effects of non-invasive cortical stimulation on skilled motor function in chronic stroke. Brain 2005;128(Pt 3):490-9.

3. Khedr EM, Etraby AE, Hemeda M, Nasef AM, Razek AA. Long-term effect of repetitive transcranial magnetic stimulation on motor function recovery after acute ischemic stroke. Acta Neurol Scand 2010;121:30-
7.

4. Ko MH, Han SH, Park SH, Seo JH. Improvement of hand function with transcranial direct current brain polarization in stroke patients. J Korean Acad Rehabil Med 2009;33:259-64.

5. Chang WH, Kim YH, Bang OY, Kim ST, Park YH, Lee PK. Long-term effects of rTMS on motor recovery in patients after subacute stroke. J Rehabil Med 2010;42:758-64.

6. Kim SY, Shin SB, Lee SJ, Kim TU, Hyun JK. Factors associated with upper extremity functional recovery following low-frequency repetitive transcranial magnetic stimulation in stroke patients. Ann Rehabil Med 2016;40:373-82.

7. Hickmott PW, Merzenich MM. Local circuit properties underlying cortical reorganization. J Neurophysiol 2002;88:1288-301.

8. Kujirai T, Caramia MD, Rothwell JC, Day BL, Thompson PD, Ferbert A, et al. Corticocortical inhibition in human motor cortex. J Physiol 1993;471:501-19.

9. Cicinelli P, Pasqualetti P, Zaccagnini M, Traversa R, Oliveri M, Rossini PM. Interhemispheric asymmetries of motor cortex excitability in the postacute stroke stage: a paired-pulse transcranial magnetic stimulation study. Stroke 2003;34:2653-8.

10. Manganotti P, Patuzzo S, Cortese F, Palermo A, Smania N, Fiaschi A. Motor disinhibition in affected and unaffected hemisphere in the early period of recovery after stroke. Clin Neurophysiol 2002;113:936-43.

11. Liepert J, Schwenkreis P, Tegenthoff M, Malin JP. The glutamate antagonist riluzole suppresses intracortical facilitation. J Neural Transm (Vienna) 1997;104:120714.

12. Shimizu T, Hosaki A, Hino T, Sato M, Komori T, Hirai $\mathrm{S}$, et al. Motor cortical disinhibition in the unaffected hemisphere after unilateral cortical stroke. Brain 2002;125(Pt 8):1896-907.

13. Rossini PM, Barker AT, Berardelli A, Caramia MD, Caruso G, Cracco RQ, et al. Non-invasive electrical and magnetic stimulation of the brain, spinal cord and roots: basic principles and procedures for routine clinical application. Report of an IFCN committee. Electroencephalogr Clin Neurophysiol 1994;91:79-92.

14. Liepert J, Hamzei F, Weiller C. Motor cortex disinhibition of the unaffected hemisphere after acute stroke. Muscle Nerve 2000;23:1761-3. 
15. Boroojerdi B, Diefenbach K, Ferbert A. Transcallosal inhibition in cortical and subcortical cerebral vascular lesions. J Neurol Sci 1996;144:160-70.

16. Netz J, Lammers T, Homberg V. Reorganization of motor output in the non-affected hemisphere after stroke. Brain 1997;120(Pt 9):1579-86.

17. Turton A, Wroe S, Trepte N, Fraser C, Lemon RN. Contralateral and ipsilateral EMG responses to transcranial magnetic stimulation during recovery of arm and hand function after stroke. Electroencephalogr Clin Neurophysiol 1996;101:316-28.

18. Boddington LJ, Reynolds JN. Targeting interhemispheric inhibition with neuromodulation to enhance stroke rehabilitation. Brain Stimul 2017;10:214-22.

19. Di Pino G, Pellegrino G, Assenza G, Capone F, Ferreri F, Formica D, et al. Modulation of brain plasticity in stroke: a novel model for neurorehabilitation. Nat Rev Neurol 2014;10:597-608.
20. Mittmann T, Luhmann HJ, Schmidt-Kastner R, Eysel UT, Weigel H, Heinemann U. Lesion-induced transient suppression of inhibitory function in rat neocortex in vitro. Neuroscience 1994;60:891-906.

21. Honaga K, Fujiwara T, Tsuji T, Hase K, Ushiba J, Liu M. State of intracortical inhibitory interneuron activity in patients with chronic stroke. Clin Neurophysiol 2013;124:364-70.

22. Lefaucheur JP, Andre-Obadia N, Antal A, Ayache SS, Baeken C, Benninger DH, et al. Evidence-based guidelines on the therapeutic use of repetitive transcranial magnetic stimulation (rTMS). Clin Neurophysiol 2014;125:2150-206.

23. Cassidy JM, Chu H, Chen M, Kimberley TJ, Carey JR. Interhemispheric inhibition measurement reliability in stroke: a pilot study. Neuromodulation 2016;19:83847. 